\title{
A Framework to assess the effect of reduction in inertia on system frequency response
}

DOI:

10.1109/PESGM.2016.7741695

\section{Document Version}

Accepted author manuscript

Link to publication record in Manchester Research Explorer

\section{Citation for published version (APA):}

Adrees, A., Papadopoulos, P., \& Milanovic, J. V. (2016). A Framework to assess the effect of reduction in inertia on system frequency response. In Power and Energy Society General Meeting (PESGM), 2016 https://doi.org/10.1109/PESGM.2016.7741695

\section{Published in:}

Power and Energy Society General Meeting (PESGM), 2016

\section{Citing this paper}

Please note that where the full-text provided on Manchester Research Explorer is the Author Accepted Manuscript or Proof version this may differ from the final Published version. If citing, it is advised that you check and use the publisher's definitive version.

\section{General rights}

Copyright and moral rights for the publications made accessible in the Research Explorer are retained by the authors and/or other copyright owners and it is a condition of accessing publications that users recognise and abide by the legal requirements associated with these rights.

\section{Takedown policy}

If you believe that this document breaches copyright please refer to the University of Manchester's Takedown Procedures [http://man.ac.uk/04Y6Bo] or contact uml.scholarlycommunications@manchester.ac.uk providing relevant details, so we can investigate your claim.

\section{OPEN ACCESS}




\title{
A Framework to Assess the Effect of Reduction in Inertia on System Frequency Response
}

\author{
Atia Adrees, Member, IEEE, Panagiotis N. Papadopoulos, Member, IEEE, Jovica V. Milanović, Fellow, IEEE \\ School of Electrical and Electronic Engineering \\ The University of Manchester \\ Manchester, UK \\ atia.adrees@manchester.ac.uk, panagiotis.papadopoulos@manchester.ac.uk, jovica.milanovic@manchester.ac.uk
}

\begin{abstract}
This paper presents a framework to analyze the impact of different penetration levels of renewable generation sources (RES) and consequent reduction in inertia on grid frequency. The developed methodology is demonstrated using three operating conditions of the network. For each operating condition, the decrease in the network loading is balanced by disconnecting a part of synchronous generation. To establish the critical penetration levels of renewables and inertia limits for the grid frequency, the uncertainty of loads, intermittent and stochastic patterns of RES generation around each operating condition are simulated.

The results clearly identify the critical penetration levels of RES and reduction in inertia limits of the system for frequency stability. In addition, the performed analysis quantifies the effect of primary frequency response and reduction in inertia on frequency nadir. The proposed framework is applied to the modified 16 machine and 68 bus network.
\end{abstract}

Index Terms - Frequency excursions, Inertia, Primary frequency response

\section{INTRODUCTION}

$\mathrm{F}$ UTURE power networks will incorporate a large number of renewable energy sources (RES) as a part of an effort towards decarbonization and diversification of energy sources. It is expected that a large proportion of RES will be based on the wind and solar generation. Renewable energy sources are characterized by their stochastic, intermittent generation patterns and by their power electronic interface with the grid. Mechanical dynamics of converter connected generation are completely decoupled from the grid $[1,2]$. The replacement of conventional synchronous generation with converter connected generation reduces system inertia and primary frequency response of the system [3-5]. In this reduced inertia system, the most challenging periods can be off-peak hours when fewer conventional synchronous generators are scheduled. During these hours system inertia is low and few power plants are available to provide frequency response services. This can have a significant impact on frequency dynamics and power system security.

It is a general practice to study the collective performance of all generators in the network for frequency stability. In such cases, all generators in the network are typically aggregated into an equivalent generator. This equivalent generator has an inertia constant equal to the sum of the inertia constants of all generators, and it is driven by the combined mechanical outputs of the individual turbines. The effects of the system loads are lumped into a single damping constant D. The speed of the equivalent generator represents the system frequency. In the case of a disturbance in one area of the system, the frequency will be different in different areas of the system due to the different electrical distance from the disturbance. Therefore, by aggregating all the generators into the single generator, some valuable information about the frequency can be lost. This, consequently, can affect the protection systems of the individual generators.

This paper presents a framework to perform a robust analysis of frequency excursion in a reduced inertia system taking into account the stochastic and intermittent patterns of renewable generation. In the network, all generators (synchronous and renewable) and associated controls are modelled using full dynamic models for generators and appropriate models for controllers. The methodology establishes the critical penetration levels of RES and inertia limits in the studied system for the grid frequency stability. The change in frequency nadir due to a reduction in system inertia is also quantified.

\section{TEST SYSTEM}

Studies are performed in modified IEEE 16 machine, 68 bus reduced order equivalent model of New England Test System and New York Power system. The network (including RES) is shown in Fig. 1. System analysis and simulations are performed within DIgSILENT PowerFactory.

Generators G1-G9 form NETS, NYPS includes G10-G13, 


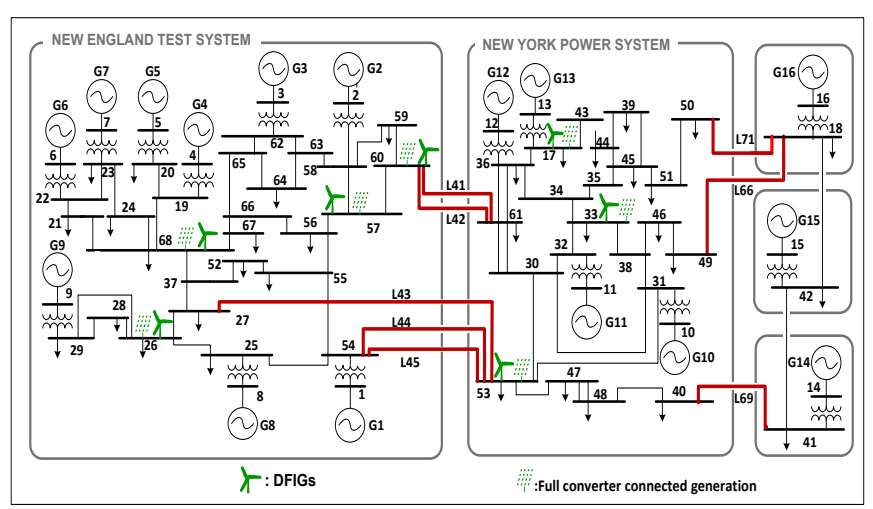

Fig. 1: IEEE 16machine network with renewables

and three neighboring areas are represented by generators G14, G15 and G16. G9 is equipped with fast-acting exciter (IEEE-ST1A) and power system stabilizer (PSS). All other generators in the network are under slow excitation (IEEEDC1A). All generators in the network include speed governor systems; generator G1 comprises GAST speed governor; G3 and G9 include IEEEG3 (hydro turbine) and G2-G8, G10G16 incorporate IEEEG1 (steam turbine). The synchronous generators are represented by sixth order models. Transmission lines are modeled with the standard $\pi$ circuit. Loads are modelled as constant impedance. Load dependence on frequency is not considered deliberately at this stage to isolate the influence of inertia reduction. Full system details, generator and exciter parameters are given in [6].

A portion $(30 \%)$ of synchronous generators in NETS and NYPS is replaced by renewables (RES); half of that, $15 \%$, comprising doubly fed induction generators (DFIGs) and another half, 15\%, full converter connected (FCC) units. RES are connected to buses 26, 68, 57 and 60 in NETS, and to buses 17, 33 and 53 in NYPS. The buses where RES are connected have been chosen randomly provided voltages, and thermal limits of the lines are not violated, and power flow through tie-lines stays close to that reported in [6].

\section{A. Type 3, DFIG Modelling}

Wind turbines are modelled as Type 3 doubly fed induction generators (DFIFs) with rotor side converter. The modelling approach is similar to two recent working groups WECC and IEC $[7,8]$.

\section{B. Type 4 Full Converter Connected (FCC) Unit Modelling}

All generator connected to the grid through full converter interface like PVs can be represented by Type 4 WTG model for system stability studies [7]. This is appropriate for system stability studies as the converter decouples the mechanical dynamics of the unit from the electric grid [9]. The model used within this research work is Type 4 WTG generic model available in DIgSILENT PowerFactory [7, 8].

\section{CASE STUdiES}

All power systems exhibit frequency excursions following an active power disturbance in the network. Frequency nadir (the minimum frequency following an active power disturbance) is governed by the system inertia and governors response. In the absence of speed governors, frequency nadir is determined by the size of active power disturbance and inertia of the system.

To establish the effect of reduction in inertia and primary frequency response on frequency nadir in a reduced inertia system following case studies are developed.

i. Nominal loading and no renewable energy sources in the network.

ii. At the nominal loading of the network, $30 \%$ of synchronous generation is replaced by renewables in two areas of the network. This reduces the inertia of the system and primary frequency response.

iii. With $30 \%$ renewables and $70 \%$ synchronous generation in the network, loading of the network is reduced to $\mathbf{6 0 \%}$. $28 \%$ reduction in the load is balanced by disconnecting synchronous generation, and remaining $12 \%$ reduction in the load by de-loading synchronous generators. This further reduces the inertia and primary frequency response and increases the nominal penetration of renewables to $46 \%$.

iv. With $30 \%$ synchronous generation replaced by renewables, loading of the network is reduced to $\mathbf{4 0 \%}$. $42 \%$ reduction in the load is balanced by disconnecting synchronous generation and $18 \%$ by de-loading generators. This reduces the inertia of the system even further, and the nominal penetration level increases to $52 \%$.

The nominal penetration level $\left(\boldsymbol{N P} \boldsymbol{L}_{\boldsymbol{a}}\right)$ of RES is defined as installed capacity ( the nominal/maximum power) of renewables based on the total generation (synchronous+renewables) in the network, and is calculated using (1)[10].

$$
N P L_{a}=\frac{\Sigma_{n=1}^{d} P_{R E S, n}^{0}}{\Sigma_{m=1}^{g} S_{S G, m} \cdot p f_{S G, m}+\Sigma_{n=1}^{d} P_{R E S, n}^{0}}
$$

Where $\boldsymbol{S}_{\boldsymbol{S G}, \boldsymbol{m}}$ is the toal synchronous generation in the network at particular operating condition, $\boldsymbol{p} \boldsymbol{f}_{\boldsymbol{S G}, \boldsymbol{m}}$ is the powerfactor of synchronous generators and $\boldsymbol{P}_{\boldsymbol{R E S}, \boldsymbol{n}}^{\mathbf{n}}$ is the nominal/ maximum power output of renewables.

Table I shows the nominal penetration level of RES for each case study and equivalent inertia of the system for each area.

TABLE I: INERTIA VALUES AND NOMINAL PENETRATION (NPL) LEVELS

\begin{tabular}{|c|c|c|c|c|c|}
\hline & \multirow{2}{*}{ NPL } & \multicolumn{4}{|c|}{ Equivalent ' $H^{\prime}$ sec } \\
\cline { 3 - 6 } & NET \& NYPS & $\boldsymbol{H}_{\text {NETS }}$ & $\boldsymbol{H}_{\text {NYPS }}$ & $\boldsymbol{H}_{E q}$ & $\boldsymbol{H}_{S y s}$ \\
\hline Case study i & 0 & 3.9 & 7.9 & 11.1 & 7.95 \\
\hline $\begin{array}{c}\text { Case study ii } \\
\text { (nominal loading) }\end{array}$ & $30 \%$ & 2.7 & 5.5 & 11.1 & 6.8 \\
\hline $\begin{array}{c}\text { Case study iii (60\% } \\
\text { loading) }\end{array}$ & $45 \%$ & 1.64 & 3.32 & 7.8 & 4.1 \\
\hline $\begin{array}{c}\text { Case study iv (40\% } \\
\text { loading) }\end{array}$ & $52 \%$ & 1.28 & 2.26 & 6.6 & 2.86 \\
\hline
\end{tabular}

Simulations including the uncertainties with RES generation and loads are performed to establish the effect of reduction in inertia with and without speed governor system. 


\section{RESULTS AND ANALYSIS}

The inertia constant of a synchronous generator defines its response to any changes in power balance. The inertia constant of a generator $(\boldsymbol{H})$ can be viewed as the time the generator can maintain full electrical power output without any mechanical power input.

The system inertia $\boldsymbol{H}_{\text {sys }}$ of 16 machine system, with nominal loading, as given in [6], is calculated using (2). Where $\boldsymbol{H}_{\boldsymbol{i}}, \boldsymbol{S}_{\boldsymbol{i}}$ and $\boldsymbol{n}$ denote the inertia constant of the each generator, the generator rating and the number of units respectively.

$$
H_{s y s}=\frac{\Sigma_{i=1}^{n} S_{i} H_{i}}{\Sigma_{i=1}^{n} S_{i}}
$$

Inertia of three areas, inertia of NETS $\boldsymbol{H}_{\text {NETS }}$, inertia of NYPS $\boldsymbol{H}_{N Y P S}$ and inertia of equivalent areas $\boldsymbol{H}_{\boldsymbol{E} \boldsymbol{q}}(\mathrm{G} 14, \mathrm{G} 15$ and G16) is also calculated.

Replacement of $30 \%$ of synchronous generation with renewables in NETS and NYPS area reduces the overall inertia $\boldsymbol{H}_{\text {sys }}$ of the system, $\boldsymbol{H}_{N E T S}$ and $\boldsymbol{H}_{N Y P S}$. Inertia constant $\boldsymbol{H}$ can be expressed in terms of moment of inertia $\boldsymbol{J}$

$$
H=\frac{J \omega_{m}^{2}}{2 V A_{\text {base }}}
$$

$\boldsymbol{J}$ is the moment of inertia of generator in $\mathrm{kg} . \mathrm{m}^{2}$ and $\boldsymbol{\omega}_{\boldsymbol{m}}$ is the angular velocity of the rotor in mech. $\mathrm{rad} / \mathrm{s}$.

Total moment of inertia $\boldsymbol{J}$ of the system is calculated for each case study case. As synchronous generation reduces in the network, total moment of inertia of synchronous generation also reduces. $\boldsymbol{H}_{\text {sys }}$ calculated using (2) is reduced in the same proportion as $\boldsymbol{J}$ is reduced in each study case.

Table I illustrates the impact of increased penetration of RES on the network inertia. It can be seen that the network has low, medium and high inertia areas in the network. $\boldsymbol{H}_{\text {NETS }}$ is only $3.9 \mathrm{~s}$ without RES and 52\% penetration of RES reduces $\boldsymbol{H}_{\text {NETS }}$ to $1.28 \mathrm{~s}$. $\boldsymbol{H}_{\text {NYPS }}$ is $7.9 \mathrm{~s}$ without RES and this reduces to $2.26 \mathrm{~s} \mathrm{(67 \%} \mathrm{reduction)} \mathrm{with} 52 \%$ RES penetration. The overall inertia of the system is $7.95 \mathrm{~s}$ and this decreases to $2.86 \mathrm{~s}$ when the penetration of RES increases to $52 \%$.

\section{A. Loading Uncertainty}

Uncertainties in the accuracy of loading forecasts are modelled by a variation in the loading values for each system bus. All loads are assumed to be normally distributed with mean nominal values and standard deviation of $3.34 \%(10 \%$ at $3 \sigma)[11]$.

\section{B. Uncertainty with RES Generation}

Uncertainties in PV farms power generation are represented by a variation in the power of each PV farm. All PV farms follow a beta distribution with $a=13.7$ and $b=1.3$. Uncertainties with wind generation are modelled by varying the output power of the wind farm. It is assumed that each wind farm follows Weibull distribution with $\alpha=2.2 \&$ $\beta=11.1[11]$.

The distributions are sampled separately for each load, wind and solar farms. An optimal load flow solution is then used to determine the corresponding output of synchronous generators.

\section{Defining critical inertia and penetration levels of RES}

As the inertia of the system reduces due to disconnection of synchronous generation and increased nominal penetration of renewables, small variations in the active power cause bigger variation in frequency nadir. In these studies, a threshold of $500 \mathrm{mHz}$ for frequency nadir variation is considered. When $\pm 10 \%$ variation in active power disturbance leads to a variation in frequency nadir greater than $500 \mathrm{mHz}$; the inertia of the system and the nominal penetration level of RES in the network are considered to be critical. Variation in active power disturbance and threshold for frequency nadir can be customized.

D. Impact of increased penetration of RES on system inertia for each studied operating condition

Modelling the stochastic and intermittent behavior of the wind and solar generation, and uncertainties in the loading forecast change the instant penetration level of RES in NETS and NYPS at each operating condition in a continuous manner. Instant penetration of renewables $P L_{i a}$ of the area is calculated as

$$
P L_{i a}=\frac{\Sigma_{n=1}^{d} P_{R E S, i n}}{\Sigma_{m=1}^{g} P_{S G, i m}+\Sigma_{n=1}^{d} P_{R E S, i n}}
$$

The subscript $i=1 \ldots 1000$ denotes the dynamic simulations number, $a=1 \ldots 5$ the system area, $g$ the generator number and $d$ the wind and solar farm number. Where $\boldsymbol{P}_{R E S, \text { in }}$ is the active power produced by the wind and solar farm in the area, $\boldsymbol{P}_{\boldsymbol{S G}, \boldsymbol{i m}}$ is the power generated by each synchronous generator in the area [10].

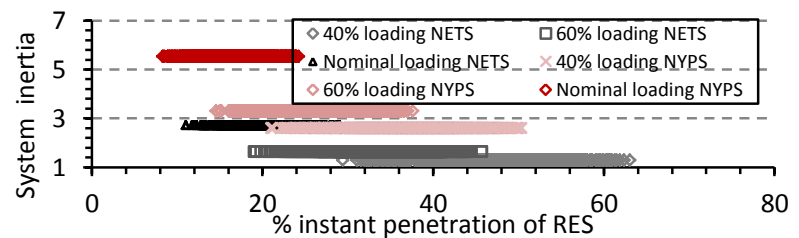

Fig. 2: Inertia of the system against \% instant penetration of RES

Simulations are performed by introducing an active power disturbance of 1340MW (a simultaneous outage of G2, G7 and G10) for each operating point. Due to uncertainties in loads and renewables, the power output of generators varies by $\pm 10 \%$. Therefore, active power disturbance varies within $\pm 10 \%$ of $1350 \mathrm{MW}$.

Frequency nadir following this active power disturbance is recorded on each bus connected to tie-line between NETS and NYPS area. Power transfer through line L41 is the highest and NYPS is importing power from NETS. Therefore, frequency nadir at bus 61 is discussed throughout this work. The system frequency following an active power disturbance at buses $60,27,54,61$ and 53 differs slightly but not significantly.

It can be seen from Fig. 2 penetration levels of RES vary between $10 \%-30 \%, 18 \%-46 \%$ and $28 \%-64 \%$ at nominal, $60 \%$ and $40 \%$ loading respectively. For the same installed capacity of RES, \% instant penetration of RES and the range of its variation increases with the increase in disconnection of 
synchronous generation.

It can also be observed from Fig. 3 that penetration levels of RES at $60 \%$ loading overlap penetration levels at nominal loading and $40 \%$ loading. However, the band of variation of frequency nadir for each operating condition is distinct.

In this analysis, the inertia of the system is constant for one operating conditions and only varies with the operating condition of the network. The small variations in the active power disturbance result in small variations in frequency nadir within one operating condition. The variation range in frequency nadir within one operating condition increases as the inertia of the system reduces.

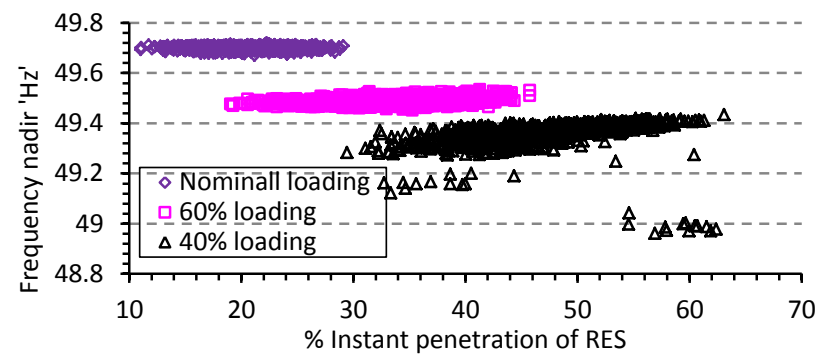

Fig. 3: Frequency nadir with three operating conditions

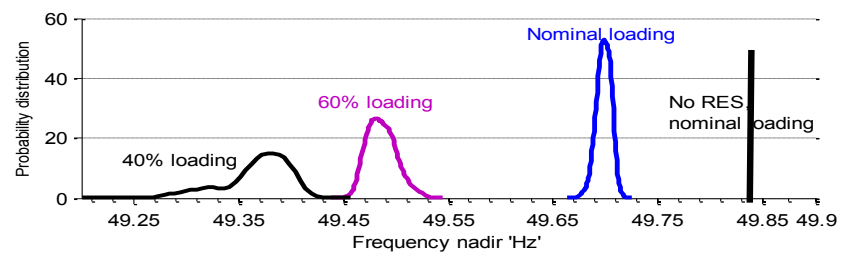

Fig. 4: $p d f s$ of frequency nadir for three studied operating conditions.

It is to be noted that the same active power disturbance results in different values of frequency nadir for the same penetration levels at different operating conditions of the network. For 30\% penetration level of RES, frequency nadir is approximately $49.67 \mathrm{~Hz}$ at the nominal loading, moves to $49.46 \mathrm{~Hz}$ at $60 \%$ loading. It further drops to $49.3 \mathrm{~Hz}$ at $40 \%$ loading. Since the number of synchronous generation varies in three studied operating conditions, the inertia of the system is different in each operating condition. The drop in frequency nadir increases as the inertia of the system reduces.

To determine the most probable values for three studied operating conditions, $p d f_{s}$ (probability density functions) of frequency nadir are plotted, shown in Fig. 4. It can be seen that frequency nadir is well within the operation limits, \pm 200 $\mathrm{mHz}$, without renewables at the nominal loading of the network. Replacement of $30 \%$ of synchronous generation with RES moved the most probable value of frequency nadir to $49.7 \mathrm{~Hz}$ and increased the drop in frequency nadir to 0.3 Hz. At $60 \%$ loading, disconnection of $40 \%$ synchronous generation shifts the mode of the $p d f$ to $49.48 \mathrm{~Hz}$, further increasing the drop in frequency nadir to $0.52 \mathrm{~Hz}$. At $40 \%$ loading, the most probable value of the $p d f$ moves to $49.38 \mathrm{~Hz}$ resulting drop in frequency to $0.62 \mathrm{~Hz}$.

It can also be observed that as the inertia of the system due to disconnection of synchronous generators decreases the $p d f_{s}$ have bigger deviation. Small changes in the operating point within the same operating condition lead to the different values of nadir.

Fig. 3 and Fig. 4 show that the range of variation in frequency nadir at $40 \%$ loading is much bigger than the range at $60 \%$ load and full load. At $40 \%$, loading frequency nadir varies between $49.45-48.8$. It is the critical inertia limit for this size of disturbance as small changes in the active power disturbance lead to a bigger $(>500 \mathrm{mHz})$ variation in frequency nadir.

For example, the statutory frequency limits for the UK system is $\pm 500 \mathrm{mHz}$. For $60 \%$ loading and $40 \%$ loading, the most probable values are outside this limit, synchronous generation in the system is increased ( SG connected) by $10 \%$ for each operating conditions. This increases the inertia of the system by $7 \%$. Simulations are performed with the same uncertainties. It can be seen from Fig. 5 that $7 \%$ increase in the inertia moves the most probable value of $60 \%$ loading to $49.55 \mathrm{~Hz}$.

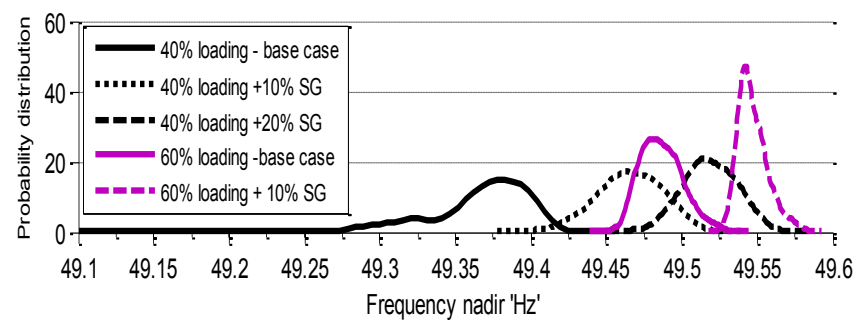

Fig. 5: $p d f s$ of frequency nadir for $60 \%$ and $40 \%$ operating

The most probable value of $40 \%$ loading $p d f$ with $10 \%$ increase in synchronous generation is $49.45 \mathrm{~Hz}$. Therefore, synchronous generation is further increased by $10 \%$; this moves the mode of the $p d f$ (thick dashed line) within the threshold bound.

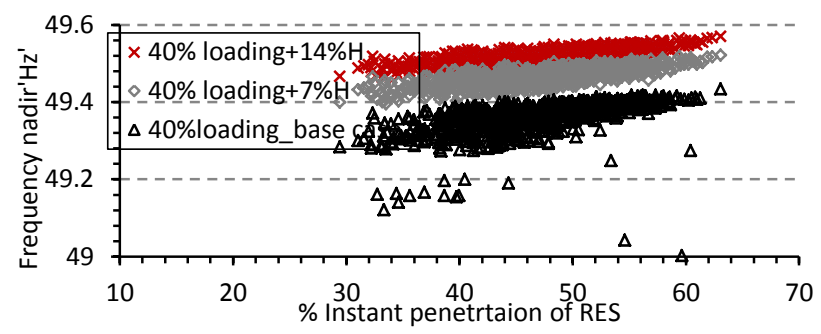

Fig. 6: Frequency of $40 \%$ loading

Fig. 6 shows frequency nadir plotted for $40 \%$ loading of the network with three values of inertia. It could be observed that for $10 \%$ increase in synchronous generation inertia, i.e., $7 \%$ increase in the system inertia, the frequency of the system is below $49.5 \mathrm{~Hz}$, but the frequency variation range is significantly reduced. Small variations in the active power disturbance do not affect frequency nadir significantly. For further $10 \%$ increase in synchronous generation, frequency nadir is within $\pm 500 \mathrm{mHz}$ limits for most of the penetration levels of RES.

It can be also observed that for the same inertia when $\%$ instant penetration of RES increases the frequency nadir improves because more power is supplied by RES and generators are relatively lightly loaded, capable of providing 
more frequency response.

It can also be concluded that when online synchronous generation is less than $50 \%$ of total generation, small variations in the active power can lead to big variation in frequency nadir; this is the critical nominal penetration level of RES for this system.

\section{E. Impact of Spinning reserves}

To investigate the effect of spinning reserves on frequency nadir, two more case studies are performed.

v. With 30\% synchronous generation replaced by renewables, loading of the network is reduced to $60 \%$. Entire $(40 \%)$ reduction in the network load is met by deloading generators. This study case has the same inertia as case study ii. However, spinning reserves are increased.

vi. With 30\% synchronous generation replaced by renewables, loading of the network is reduced to $40 \%$. $28 \%$ reduction in the network load is balanced by disconnecting synchronous generation and $42 \%$ reduction in load by de-loading generators. This study case has the same inertia as case study iii. However, the system has more spinning reserves.

In Fig. 7 case studies ii and $\mathrm{v}$ have the same inertia. However, spinning reserves in case study $\mathrm{v}$ are increased by $200 \%$. Case studies iii and vi have the same inertia, but case vi has $325 \%$ more spinning reserves due to the de-loading of the network. The inertia of the system is higher in case studies (ii, v ) than the case studies (iii, vi).

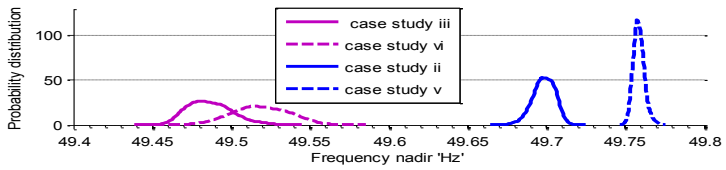

Fig. 7: $p d f s$ of frequency nadir for $60 \%$ loading and $40 \%$

It could be observed that the most probable value for the same inertia of the system has improved by $(0.06 \mathrm{~Hz})$ when the spinning reserves are increased from (1500MW to 3000 (nearly 200\% increase). In $p d f$ iii and vi the system has same inertia, spinning reserves are increased from (1000MW to $3350 \mathrm{MW}$ (increased by $325 \%$ ), however, the improvement in frequency nadir is $0.05 \mathrm{~Hz}$.

\section{$F$. Impact of change in inertia}

The case studies ii, iii and iv are performed without governors.

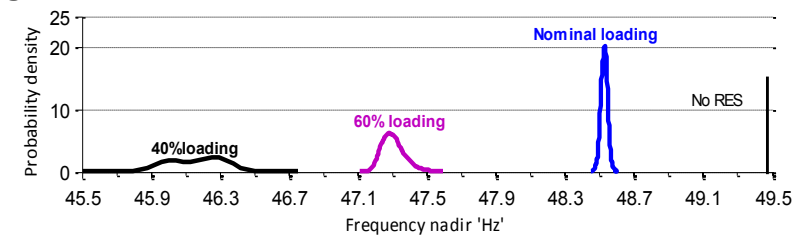

Fig. 8: $p d f s$ of frequency nadir for three operating conditions without governors

It can be observed that $30 \%$ reduction in inertia moves the most probable value from $49.48 \mathrm{~Hz}$ to $48.5 \mathrm{~Hz}$ resulting in $0.98 \mathrm{~Hz}$ increase in frequency nadir. Further disconnection of synchronous generation at $60 \%$ loading reduces inertia by $48 \%$. This shifts the mode of the $p d f$ to $47.3 \mathrm{~Hz}$. At $40 \%$ loading, $67 \%$ reduction in the inertia of the system shifts the most probable value to $46.1 \mathrm{~Hz}$. Fig. 9 shows the increase in the drop of frequency nadir with the reduction in the system inertia that is linear.

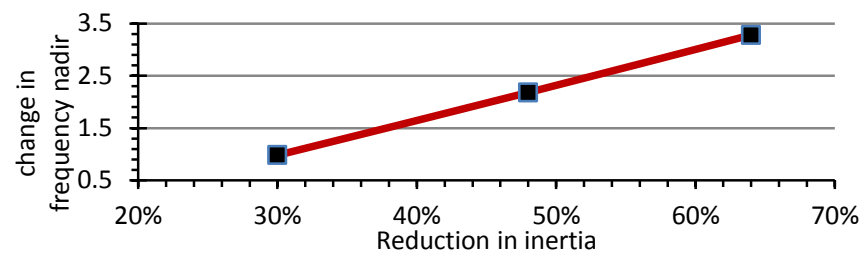

Fig. 9: change in frequency nadir with the reduction in inertia in the system

\section{CONCLUSIONS}

This paper presented an approach to perform a robust analysis of the effect of reduction in inertia and primary response of frequency excursions in a large system. The analysis successfully established the reduction in inertia limits and the critical nominal penetration level of RES for the grid frequency in the test system. The results established when nominal penetration level of RES generation is higher than the online synchronous generation; small changes in the active power disturbance can lead to much bigger variation in frequency nadir.

The performed analysis also shows that amount of spinning reserves required to reduce the drop in frequency nadir increases as the inertia of the system decreases.

\section{REFERENCES}

[1] J. F. Conroy and R. Watson, "Frequency Response Capability of Full Converter Wind Turbine Generators in Comparison to Conventional Generation," IEEE Transactions on Power Systems, vol. 23, pp. 649656, 2008

[2] Brian Parsons, Michael Milligan, Bob Zavadil, Daniel Brooks, Brendan Kirby, Ken Dragoon, and Jim Caldwell, "Grid impacts of wind power: a summary of recent studies in the United States," Wind Energy, vol. 7: John Wiley \& Sons Ltd., 2004.

[3] T. Weissbach and E. Welfonder, "High-frequency deviations within the European Power System: Origins and proposals for improvement," in IEEE Power Systems Conference and Exposition, 2009.

[4] J. W. Ingleson and D. M. Ellis, "Tracking the Eastern interconnection frequency governing characteristic," in IEEE PES General Meeting, 2005.

[5] I. Erlich, K. Rensch, and F. Shewarega, "Impact of large wind power generation on frequency stability," in IEEE PES General Meeting, 2006.

[6] P. Pal and B. Chauduri, Robust Control in Power Systems. New York: Springer Science \& Business Media, 2005.

[7] WECC Renewable Energy Modeling Task Force, "WECC Solar Plant Dynamic Modeling Guidelines" 2014.

[8] P. Sorensen, B. Andresen, J. Fortmann, and P. Pourbeik, "Modular structure of wind turbine models in IEC 61400-27-1," in IEEE PES General Meeting, 2013.

[9] S. Eftekharnejad, V. Vittal, G. T. Heydt, B. Keel, and J. Loehr, "Impact of increased penetration of photovoltaic generation on power systems," IEEE Transactions on Power Systems, vol. 28, pp. 893-901, 2013.

[10] L. Changling, et al., "Estimation of Wind Penetration as Limited by Frequency Deviation," IEEE Transactions on Energy Conversion,, vol. 22, pp. 783-791, 2007.

[11] R. Preece and J.V. Milanovic, "Risk-Based Small-Disturbance Security Assessment of Power Systems," IEEE Transactions on Power Delivery, vol. 30, pp. 590-598, 2015. 